\title{
Packet Delay Metrics for IEEE 802.11 Distributed Coordination Function
}

\author{
P. Raptis $\cdot$ V. Vitsas $\cdot$ K. Paparrizos
}

Published online: 5 December 2008

(C) Springer Science + Business Media, LLC 2008

\begin{abstract}
In this paper, we introduce a comprehensive packet delay analysis for wireless networks based on IEEE 802.11 Distributed Coordination Function (DCF). We develop mathematical models that calculate a set of packet delay metrics, namely a) the average packet delay for successfully transmitted packets, b) the average packet delay of successfully transmitted packets experiencing a specific number of collisions, c) the average packet drop time, d) the delay jitter and e) the delay distribution by computing the probability of a packet to be successfully transmitted experiencing delay time lower than a given value. All the developed models are based on calculating station's delay time at the transmission slot(s) plus the average time that station defers at backoff slots before successful transmission. The mathematical models are simple, computationally fast and can be used to build admission control algorithms. Simulation results show that our proposed mathematical analysis is highly accurate.
\end{abstract}

Keywords wireless networks · 802.11 DCF · delay performance

\footnotetext{
P. Raptis $(\varangle) \cdot$ V. Vitsas

Department of Information Technology,

Technological Educational Institution,

Thessaloniki, Greece

e-mail: praptis@it.teithe.gr

V. Vitsas

e-mail: vitsas@it.teithe.gr

\section{K. Paparrizos}

Department of Applied Informatics, University of Macedonia,

Thessaloniki, Greece

e-mail: paparriz@uom.gr
}

\section{Introduction}

Wireless Local Area Networks (WLANs) are gaining great popularity and are getting rapidly deployed all over the world. The WLANs are flexible and easy to implement as no cables are required. The dominating protocol utilized by WLANs is the IEEE 802.11. IEEE 802.11 defines Medium Access Control (MAC) and Physical Layer (PHY) specifications for WLANs [1]. IEEE 802.11 MAC protocol is based on the carrier sense multiple access with collision avoidance (CSMA/CA) scheme and includes two modes for channel access i.e. the mandatory Distributed Coordination Function (DCF) and the optional Point Coordination Function (PCF). In DCF, there is no differentiation mechanism between delay sensitive real-time traffic (e.g. voice over IP, video) and delay insensitive traffic (e.g. ftp, e-mail); all stations compete for the channel with the same priority. PCF is a polling-based contention free access scheme that uses an Access Point (AP) as point coordinator; it is designed to support multimedia applications. DCF describes two techniques to transmit data packets; a two-way handshaking (DATA-ACK) called basic access and an optional four-way handshaking (RTS-CTS-DATA-ACK) called Request-To-Send/Clear-To-Send (RTS/CTS) access method. In the basic access, the transmitter sends a data packet (DATA) and the receiver responds with an acknowledgement (ACK) after the successful reception of the data packet. The RTS/CTS mode requires first the exchange of short frames between the transmitter (sends a RTS frame) and the receiver (answers with a CTS frame) in order to reserve the medium before the long data packet transmission.

For real time applications, packet delay is a key issue that has to be considered when addressing performance in WLANs. Real-time applications over WLANs require that each packet arrives at the destination within a specific time 
period. If the packets do not arrive on time and some packets have high delay times, then the quality of the provided services will degrade. Packet delay metrics such as average delay, jitter and delay distribution can be computed and utilized to manage the resources and to guarantee quality of services (QoS) in a WLAN.

In this paper we present a comprehensive packet delay analysis that develops models for the following metrics a) average packet delay, b) average packet delay per stage, c) average packet drop time, d) delay jitter, and e) delay distribution. The main contributions of this paper is that (a) we present a complete set of packet delay metrics which are good indicators for the performance of IEEE 802.11 WLANs, (b) all models are accurate and simple based on station's delay time at the transmission slot plus the average time that the station defers at backoff slots before successful transmission, (c) the computational efficiency of all models is remarkable, especially the delay distribution model that involves computation of many delay values provides results in tenths of milliseconds.

The paper is organized as follows. Section 2 reviews the related work and Section 3 briefly describes the DCF access scheme of the IEEE 802.11 MAC protocol. Section 4 presents the mathematical modeling for DCF and the delay metrics analysis. Section 5 validates the accuracy of the developed models by comparing analytical with simulation results. Section 6 presents analytical results that evaluate the WLAN performance under different network scenarios and parameter settings, and, finally, Section 7 presents the conclusions and future research directions.

\section{Related work}

A considerable number of researchers worldwide show a growing interest in optimizing WLAN performance by modeling the IEEE 802.11 DCF and study performance indicators such as throughput [2-7], average packet delay [7-11], delay jitter [9, 13], packet delay distribution [1418], average packet drop time [8, 11], and average packet delay per stage [12]. Bianchi [2] proposed a Markov chain model to evaluate the performance of the DCF under an error-free channel. The key assumption of the model in [2] is that at each transmission attempt and regardless of the number of retransmissions suffered, the packet collides with a constant probability. Wu [3] modified Bianchi's Markov chain to calculate the throughput taking into account the packet's retransmission limit as specified in the standard. Chatzimisios [8] employed Wu's Markov chain to develop a simple mathematical model that calculates the average packet delay but his model has an inaccuracy as the transmission slot(s) time duration is considered equal to the average time duration of defer slots. Vukovic identified this inaccuracy in Chatzimisios model at the transmission slot of a station and proposed in [10] an analytical model for calculation of the average packet delay that makes a distinction between the defer slots and the transmission slot. However, Vukovic model has an inaccuracy in the calculation of the average time duration of defer slots. Defer slot duration is calculated for $n$ contending stations and includes the station that defers i.e. the average defer slot time is computed with an additional station ( $n$ instead of $n-1)$. Vukovic model gives accurate results when the number of stations $n$ in a network is more than 20. For networks with a small number of stations the results are overestimated, as the average defer slot time is calculated with an additional station. In [9] a jitter analysis is presented for saturated ad hoc wireless networks using the first two moments (average and variance) of the service time for a linearized model of [2]. In [13] a packet delay variance (jitter squared) analysis based on the average packet delay per stage and the probability of successful transmission per stage is used to measure the effective capacity and the achievable throughput of wireless networks. In [14] and [15] the delay distribution was studied using z-transform of the packet delay. The method employed in [14] and [15] requires long computer run times. A less complex method than $[14,15]$ is proposed in [16] that computes the probability of a packet to be successfully transmitted with delay time lower than a given value. The method in [16] still involves a number of computationally expensive operations. An effective, accurate and less complex model than [14-17] is presented in [18] that calculates the probability that a packet is successfully transmitted with a delay time equal to a given value.

In this paper a new method is introduced that calculates analytically DCF delays based on a station's delay time at the transmission attempt(s) plus the average time that station defers at backoff slots before successful transmission. Defer slot time calculation enables the development of accurate, simple, and computationally fast models, and at the same time, allows the development of analytical models that calculate a complete set of packet time delay metrics. We present simple and accurate average packet delay per stage [12] and average packet delay [11] models and we extend these models to develop (a) a novel model that computes the delay jitter and (b) a novel and simpler than [14-18] delay distribution model that computes the probability that a successfully transmitted packet experiences delay lower than a given value. A comparison of analytical results of our jitter model to that of [13] shows that our model produces results which are closer to simulation results. The jitter model developed in [13] calculates the average delay at each backoff stage while our jitter model calculates the average delay that a packet experiences at 
each backoff slot. Moreover, our model that calculates the average packet delay eliminates the inaccuracy of the model in $[10]$.

\section{Distributed coordination function}

When a wireless station implementing DCF receives a transmit request from the upper layer, it senses the channel. If the channel is idle for a period of Distributed Inter Frame Spacing (DIFS), the station proceeds with its transmission. If the channel is busy, the station defers until an idle DIFS is detected and then generates a random backoff interval before transmitting in order to minimize collision probability. The backoff time counter is decreased in terms of slot time as long as the channel is sensed idle. The counter is stopped when the channel is busy and resumed when the channel is sensed idle again for more than DIFS. The station transmits the packet when its backoff timer reaches zero. If the destination station successfully receives the packet, it waits for a short inter-frame space (SIFS) time interval and transmits an acknowledgement (ACK) packet. If the transmitting station does not receive an ACK packet within a specified ACK timeout interval, the data packet is assumed to have been lost and the station schedules a retransmission. Each station holds a retry counter that is increased by one each time the data packet is unsuccessfully transmitted. If the counter reaches the retransmission limit $R$ the packet is discarded.

The backoff time counter is chosen uniformly in the range $\left[0, W_{j}-1\right]$, where $j$ is the backoff stage (i.e. the number of packet's transmission retries) $j \in[0, R]$ and $W_{j}$ is the current contention window $(\mathrm{CW})$ size. The contention window at the first transmission of a packet is set equal to minimum value $W_{0}=W$. After an unsuccessful packet transmission, the contention window $C W$ is doubled up to a maximum value $2^{m} W$ (where $m$ is the number of $C W$ sizes). Once $C W$ reaches maximum value it remains in this value until it is reset. The $C W$ is reset to minimum after a successful packet transmission or if the packet's retransmission limit $R$ is reached

$C W= \begin{cases}2^{j} W & \text { for } 0 \leq j<m \\ 2^{m} W & \text { for } m \leq j \leq R\end{cases}$

In order to reduce the collision duration when large data packets are transmitted and to battle the hidden terminal problem the RTS/CTS access scheme is used. The RTS/ CTS follows the same backoff rules as the basic access. The station sends a short RTS packet first instead of the data packet. The receiving station responds with a CTS packet after a SIFS time interval. The sender is allowed to transmit the data packet only if it receives a valid CTS. Upon the successful reception of the data packet the receiver transmits an ACK frame. If the source station does not receive a CTS frame, the retry counter is increased by one. As the RTS and CTS packets include the duration of the ongoing transmission, the stations receiving RTS or CTS update their Network Allocation Vector (NAV) and do not transmit for the specified time period.

\section{Mathematical modeling}

This study assumes that (i) hidden terminals and capture effect conditions are not present, (ii) the channel is error-free, (iii) the contending stations are of fixed number $n$, and (iv) each station has always a packet available for transmission (saturation conditions) of the same fixed size.

\subsection{DCF modeling}

Let $b(t)$ and $s(t)$ be the stochastic processes representing the backoff time counter and the backoff stage $(0, \ldots R)$ respectively for a given station at time $t$. We utilize the same discrete-time Markov chain with [3] in order to model the bi-dimensional process $\{s(t), b(t)\}$. The key approximation in this model is that each packet collides with constant and independent probability $p$. Let $b_{j, i}=$ $\lim P\{s(t)=j, b(t)=i\}$ be the stationary distribution of ${ }_{\text {the }}^{t \rightarrow}$ Markov chain, where $j \in[0, R], i \in\left[0, W_{j}-1\right]$. The probability $\tau$ that a station transmits a packet in a randomly chosen slot time can be expressed as [3]:

$$
\begin{aligned}
\tau= & \frac{2 \cdot(1-2 p) \cdot\left(1-p^{R+1}\right)}{W \cdot\left(1-(2 p)^{m+1}\right) \cdot(1-p)+(1-2 p)} \\
& \cdot\left[\left(1-p^{R+1}\right)+W \cdot 2^{m} \cdot p^{m+1} \cdot\left(1-p^{R-m}\right)\right] \\
& \text { for } \mathrm{R}>\mathrm{m}
\end{aligned}
$$

The probability $p$ that a transmitted packet encounters a collision is given by:

$p=1-(1-\tau)^{n-1}$

Equations (2) and (3) represent a non-linear system with two unknown $\tau$ and $p$, which can be solved using numerical methods and has a unique solution.

\subsection{Average delay per stage}

The delay for a successfully transmitted packet is defined as the time interval from the time the packet is at the head of its MAC queue ready to be transmitted, until an acknowledgement for this packet is received. If a packet reaches the 
specified retry limit then this packet is dropped and its time delay is not included in the calculation of the average packet delay.

Let $E\left[D_{j}\right]$ be the average delay of successfully transmitted packets from the $j$ backoff stage. The delay $D_{j}$ is the sum of the delay times that a packet experiences at $0,1, \ldots, j$ stages and $E\left[D_{j}\right]$ is calculated as follows:

$E\left[D_{j}\right]=T_{s}+j \cdot T_{c}+E[s l o t] \sum_{i=0}^{j}\left(\frac{W_{i}-1}{2}\right)$ for $0 \leq j \leq R$

where $\left(W_{i}-1\right) / 2$ is the average number of slot times that the station defers in the $i$ stage, $T_{c}$ is the time duration the channel is sensed busy during a collision and $j T_{c}$ is the time that the packet utilizes in collisions until reaches the $j$ stage, $T_{s}$ is the time to transmit successfully from the $j$ stage, and $E[$ slot $]$ is the average time that a station defers in a slot and is given by:

$E[$ slot $]=\left(1-P_{t r}\right) \cdot \sigma+P_{t r} \cdot P_{s} \cdot T_{s}+P_{t r} \cdot\left(1-P_{s}\right) \cdot T_{c}$

where $\sigma$ is the period of an empty slot, $T_{s}$ is the time duration the channel is sensed busy during a successful transmission, $P_{t r}$ is the probability that at least one station out of $n-1$ transmits in the considered slot time and is given by:

$P_{t r}=1-(1-\tau)^{n-1}$

and $P_{S}$ is the probability that a transmission occurring on the channel is successful and is given by the probability that only one station transmits of the $n-1$ remaining stations, with the condition that a transmission occurs on the channel:

$P_{s}=\frac{(n-1) \cdot \tau \cdot(1-\tau)^{n-2}}{P_{t r}}$

The time duration of $T_{s}$ and $T_{c}$ depends upon the access method employed. For the basic access method, we have:

$T_{s}^{\text {bas }}=T_{c}^{\text {bas }}=O^{\text {bas }}+(l / C)$

where $l$ is the packet length, $C$ is the channel bit rate and $O^{b a s}$ is the data packet overhead

$O^{\text {bas }}=D I F S+T_{H}+S I F S+T_{A C K}+\delta$

and for the RTS/CTS access method:

$T_{s}^{R T S}=O^{R T S}+(l / C)$

$T_{c}^{R T S}=D I F S+T_{R T S}+S I F S+T_{C T S}$ where $O^{r t s}$ is the RTS packet overhead

$O^{R T S}=D I F S+T_{H}+T_{R T S}+3 S I F S+4 \delta+T_{C T S}+T_{A C K}$

where $T_{H}, T_{R T S}, T_{C T S}, T_{A C K}$ represent the transmission time for the header (equal to the sum of MAC and physical header), RTS, CTS, ACK, respectively, and $\delta$ is the propagation delay.

Let $Q_{j}$ be the probability that a successfully transmitted packet is transmitted successfully from the $j$ stage (probability per stage), so we get:

$Q_{j}=\frac{(1-p) p^{j}}{1-p^{R+1}}$ for $0 \leq j \leq R$

where $(1-p)$ is the probability that a packet is successfully transmitted after the packet reached the $j$ stage (after $j$ collisions) with probability $p^{j}$, provided that the packet is not dropped $\left(1-p^{R+1}\right)$.

The average packet delay per stage calculated by Eq. 4 and the probability per stage calculated by Eq. 13 are key metrics as (a) give an insight view of internal mechanisms of the DCF that affect packet delay and (b) can be used as a basis to calculate jitter, delay distribution, average delay, and average packet drop time.

\subsection{Average packet drop time}

Average packet drop time is defined as the average time that a packet is discarded after the packet reaches the retransmission limit $R$. From Eq. 4 we easily derive the average drop time:

$E\left[T_{\text {drop }}\right]=(R+1) \cdot T_{c}+E[$ slot $] \cdot \sum_{i=0}^{R}\left(\frac{W_{i}-1}{2}\right)$

where $(R+1) T_{c}$ is the time that the packet utilizes in $(R+1)$ collisions.

After some algebra (14) becomes:

$$
\begin{aligned}
E\left[T_{\text {drop }}\right]= & (R+1) \cdot T_{c} \\
& +\frac{1}{2}\left(W\left(2^{m+1}-1\right)+2^{m} W(R-m)-(R+1)\right) \\
& \cdot E[\text { slot }]
\end{aligned}
$$

\subsection{Average packet delay}

Using the developed analytical model in Section 4.2 for the average packet delay per stage $E\left[D_{j}\right]$ and the probability per 
stage $Q_{j}$, we can calculate the average packet delay $E[D]$ by:

$E[D]=\sum_{j=0}^{R}\left(E\left[D_{j} \cdot Q_{j}\right]\right)$

Substituting (4) and (13) to (16) we get:

$E[D]=\sum_{j=0}^{R}\left(\left(T_{s}+j T_{c}+E[s l o t] \sum_{i=0}^{j} \frac{W_{i}-1}{2}\right) \frac{p^{j}(1-p)}{1-p^{R+1}}\right)$

Using (18) and after some algebra, equation (17) becomes:

Let $A_{i}, B_{i}$ and $H$ be

$$
A_{i}=\frac{p\left(1-p^{i}-i \cdot p^{i}(1-p)\right)}{(p-1)^{2}}, \quad B_{i}=\frac{1-p^{i+1}}{1-p}, H=\frac{1-(2 p)^{m+1}}{1-2 p}
$$

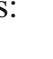

$$
\begin{aligned}
E[D]= & T_{s}+T_{c} \frac{A_{R}}{B_{R}}+\frac{1}{2 B_{R}} E[\operatorname{slot}]\left(\left(W 2^{m+1}-W-m-1\right)\left(B_{R}-B_{m}\right)-\left(A_{m}-B_{m}\right)\right. \\
& \left.+W\left(2 H-B_{m}\right)+\left(W 2^{m}-1\right)\left(A_{R}-A_{m}-m B_{R}-m B_{m}\right)\right)
\end{aligned}
$$

\subsection{Jitter}

The jitter is defined as the possible variation of the packet delay values from the mean value. Jitter (standard deviation) and variance are the most common measures of statistical dispersion (variability) from the mean.

The delay $D_{0, i}$ of a packet transmitted successfully from stage 0 and have selected the backoff value of $i$ at stage 0 is

$D_{0, i}=T_{s}+k_{s} T_{s}+k_{c} T_{c}+\left(i-k_{s}-k_{c}\right) \sigma$

where $k_{s}\left(k_{c}\right)$ is the number of successful (collided) transmissions the station encounters (from other stations) before transmitting. Because of the random nature of selection of backoff value at all stations the calculation of $k_{s}$ and $k_{c}$ for each successfully transmitted packet is complex and time consuming especially at higher stages.

The key assumption of the proposed jitter analysis (and the delay distribution analysis of next section) is that delay variation mainly originates from different backoff values selected at different stages and not from different time variations of backoff slots $\left(T_{s}, T_{c}, \sigma\right)$. This approximation greatly reduces the computational complexity, as a result, increases computational efficiency and leads to accurate analytical results. Thus, instead of computing the delay $D_{0, i}$ we compute the average delay $E\left[D_{0, i}\right]$ of packets transmitted successfully from stage 0 and have selected the backoff value of $i$ at stage 0 , considering that stations defer at each backoff slot on average time equal to $E[$ slot $]$

$E\left[D_{0, i}\right]=T_{s}+i \cdot E[$ slot $]$

$E\left[U_{0, i}\right]=T_{c}+i \cdot E[$ slot $] \quad$ for $\quad 0 \leq i \leq W_{0}-1$ where $E\left[U_{0, i}\right]$ is the average delay of packets transmitted unsuccessfully from stage 0 and have selected the backoff value of $i$ at stage 0 .

The average delay $E\left[U_{0}\right]$ of packets transmitted unsuccessfully from stage 0 is calculated using Eq. 22:

$E\left[U_{0}\right]=\frac{1}{W} \cdot \sum_{i=0}^{W-1} E\left[U_{0, i}\right]=T_{c}+E[$ slot $] \cdot \frac{W-1}{2}$

Thus, the average delay $E\left[U_{j}\right]$ of the unsuccessfully transmitted packets from stage $j$ is given by:

$E\left[U_{j}\right]=(j+1) \cdot T_{c}+E[$ slot $] \cdot \sum_{i=0}^{j}\left(\frac{W_{i}-1}{2}\right)$

for $0 \leq j \leq R$

Using Eq. 21 and Eq. 23 we can compute the average delay $E\left[D_{1, i}\right]$ of successfully transmitted packets from stage 1 (after a collision at stage 0 ):

$$
\begin{aligned}
& E\left[D_{1, i}\right]=T_{s}+i \cdot E[\text { slot }]+E\left[U_{0}\right] \\
& \quad \text { for } 0 \leq i \leq W_{1}-1
\end{aligned}
$$

Finally, the average packet delay $E\left[D_{j, i}\right]$ for packets successfully transmitted from stage $j$ and have selected the backoff value of $i$ at stage $j$ is given by:

$$
\begin{gathered}
E\left[D_{j, i}\right]=T_{s}+i \cdot E[\text { slot }]+E\left[U_{j-1}\right] \\
\text { for } 0 \leq j \leq R, 0 \leq i \leq W_{j}-1
\end{gathered}
$$

where $E\left[U_{-1}\right]=0$

The probability of a station to select backoff value $i$ at stage $j$ is $1 / W_{j}$ (independent of $i$ ) because the station utilizes random selection using a uniform distribution in the range $\left[0, W_{j}-1\right]$. Thus, the probability $P_{j}$ that a packet is successfully transmitted (provided that the packet is not 


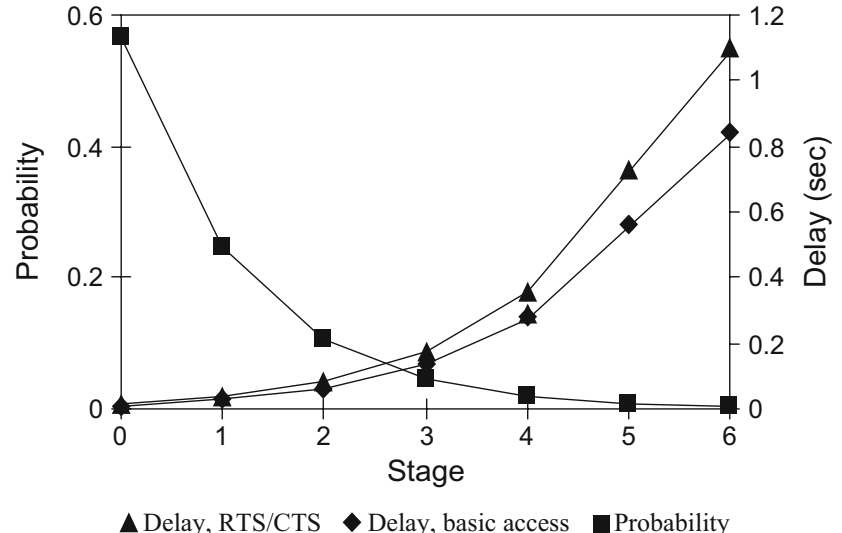

Figure 1 Average packet delay per stage and probability per stage for $n=25$

dropped) from stage $j$ selecting backoff value $i$ at the $j$ stage is:

$P_{j}=\frac{(1-p) p^{j}}{1-p^{R+1}} \frac{1}{W_{j}} \quad$ for $\quad 0 \leq j \leq R$

Now, we can compute $E\left[D^{2}\right]$ that is the average of the squared delays:

$E\left[D^{2}\right]=\sum_{j=0}^{R}\left(\sum_{i=0}^{W_{j}-1}\left(E\left[D_{j, i}\right]\right)^{2} \cdot P_{j}\right)$

Substituting (26) and (27) in (28) we get:

$E\left[D^{2}\right]=\sum_{j=0}^{R} \frac{(1-p) p^{j}}{1-p^{R+1}} \frac{1}{W_{j}} \sum_{i=0}^{W_{j}-1}\left(T_{s}+i \cdot E[\text { slot }]+E\left[U_{j-1}\right]\right)^{2}$

where $E\left[U_{-1}\right]=0$

Using (17) and (29) we can compute the jitter $J$ of the packet delays:

$J=\sqrt{E\left[D^{2}\right]-(E[D])^{2}}$

\subsection{Packet delay distribution}

Let $D$ be the set of packet delay values that are obtained from Eq. 26 and $P_{D}$ be the set of corresponding probability values obtained from Eq. 27. The cumulative distribution function $(c d f)$ that computes the probability of a packet to be successfully transmitted with delay time lower than a given value $d$ is:

$P(D<d)=\sum_{d_{i}<d} P_{D}\left(d_{i}\right) \quad$ for $1 \leq i \leq h$

where $d_{i}$ is the delay value in $i$ position of set $D$ and $h$ is the number of pair values $\left(d_{i}, P_{D}\left(d_{i}\right)\right)$ of delay and probability; $h$ is equal to the sum of backoff slots plus transmission slots:

$h=\sum_{j=0}^{R} 2^{j} W=\left(2^{m+1}-1\right) W+(R-m) 2^{m} W$

\section{Validation}

The proposed models are validated by comparing their analytical results with simulation outcome. All simulation results for all proposed models are taken with a $95 \%$ confidence interval lower than 0.007 . The parameter values used for both simulation and analytical results follow the values specified for the Direct Spread Sequence Spectrum (DSSS) employed in the IEEE $802.11 \mathrm{~b}$ standard and are shown in Table 1. Unless otherwise specified, all stations use a constant data packet length of $l=8,184$ bits transmitted at $C=11 \mathrm{Mbit} / \mathrm{s}$.

Figure 1 plots probability per stage and average packet delay per stage for basic access and RTS/CTS access scheme. Figure indicates that the models for average packet delay per stage $\left(E\left[D_{j}\right]\right.$ Eq. 4$)$ and for probability per stage $\left(Q_{j}\right.$ Eq. 13) are accurate as the analytical results (lines) match the simulation results (symbols) for both access methods. The figure shows that the probability per stage for successful packet transmission lowers as the backoff stage gets higher because more collisions are needed to reach higher stages and is independent of the access method employed (basic or RTS/CTS).

Figure 2 plots average packet delay $(E[D]$ Eq. 19) and average packet drop time ( $E\left[T_{d r o p}\right]$ Eq. 15$)$ versus number of stations for basic and RTS/CTS access. Figure shows that analytical results (lines) match simulation results (symbols) in both access methods. As number of stations increases, the number of collisions is increased resulting in

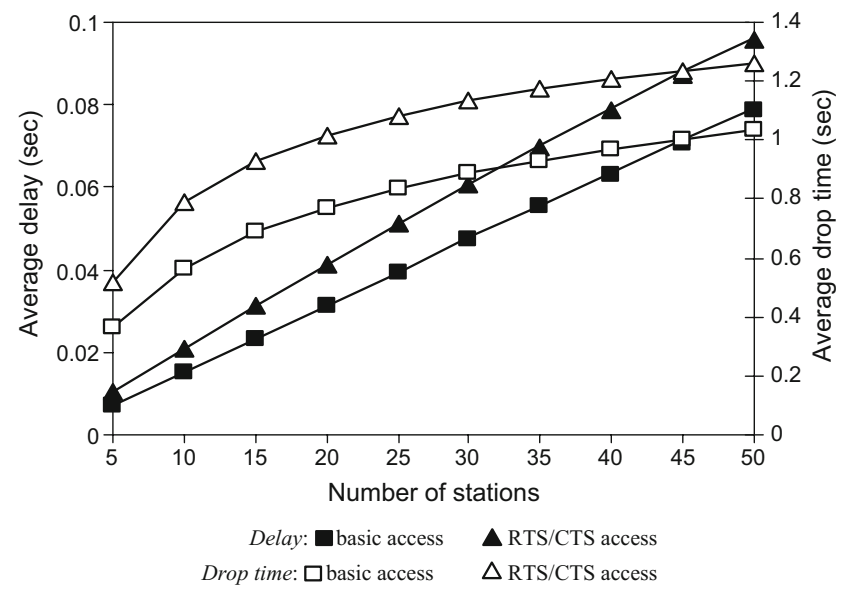

Figure 2 Average packet delay and average drop time versus number of stations 
higher delays. Although the number of collisions is high when the network size increases, the basic access scheme achieves lower average packet delay and packet drop time values compared to the RTS/CTS handshake mechanism. This can be explained by considering that the exchange of the RTS and CTS reservation packets at a much lower control rate, results in more significant delays in communication than the delays of long data packet collisions in basic access.

Figure 3 validates the delay jitter model (Eq. 30) by plotting analytical and simulation results of delay jitter versus number of stations for basic and RTS/CTS access schemes. We further compare results using our jitter model developed in Section 4.4 against the model developed in [13] and show that the results of our jitter model are closer to simulation results for both access schemes. The figure also indicates that large network sizes result in higher jitter. This can be explained by considering that as the number of stations increases, more transmitted packets collide and, thus, stations utilize higher stages for packet transmission. Packets successfully transmitted from higher stages are expected to have higher delay times and, thus, the packet delay variability is increased.

Figures 4 and 5 illustrate analytical and simulation results of cumulative delay distribution-i.e. $P(D<d)$ versus $d$ (Eq. 32)-for basic and RTS/CTS access respectively. Results show that the delay distribution model is fairly accurate as there is a good match between the analytical and simulation results for both access modes. A closer look at Figs. 4 and 5 also confirms that the RTS/CTS access attains higher packet delay values compared to basic access scheme. Within specific period of time more packets are transmitted with basic access than with RTS/CTS scheme as the packet transmission in RTS/CTS access takes longer time than in basic access. In order to

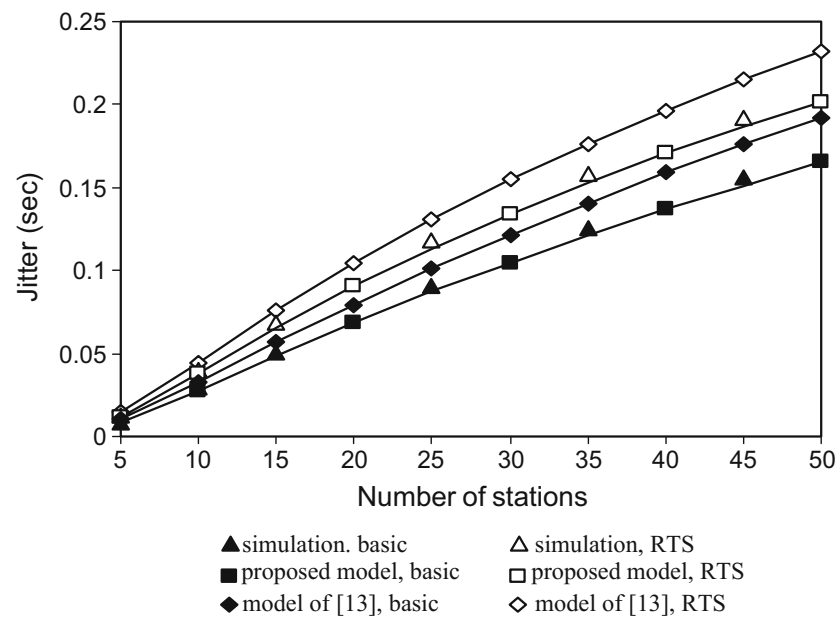

Figure 3 Packet delay jitter versus number of stations

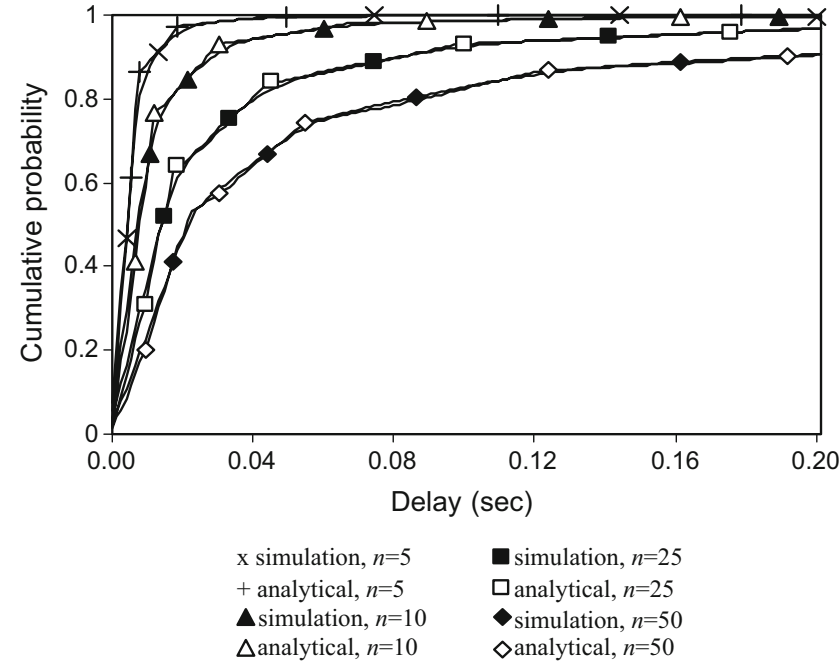

Figure 4 Cumulative delay distribution for basic access mode and different network sizes

understand the computational efficiency of our model, we measured the times required to perform the computation. We verified that our model computes all values of a cumulative distribution curve in less than $21 \mathrm{~ms}$ in a PC Pentium $3 \mathrm{GHz}$ using Matlab7 interpreter, that is 3.5 times faster than the model presented in [18].

\section{Performance evaluation}

The following analytical results are obtained using the parameter values presented in Table $1, C=11 \mathrm{Mbit} / \mathrm{s}, l=$ $8,184 \mathrm{bits}$, and fixed number of contending stations $n$ (unless otherwise specified). When channel bit rate is $C=$

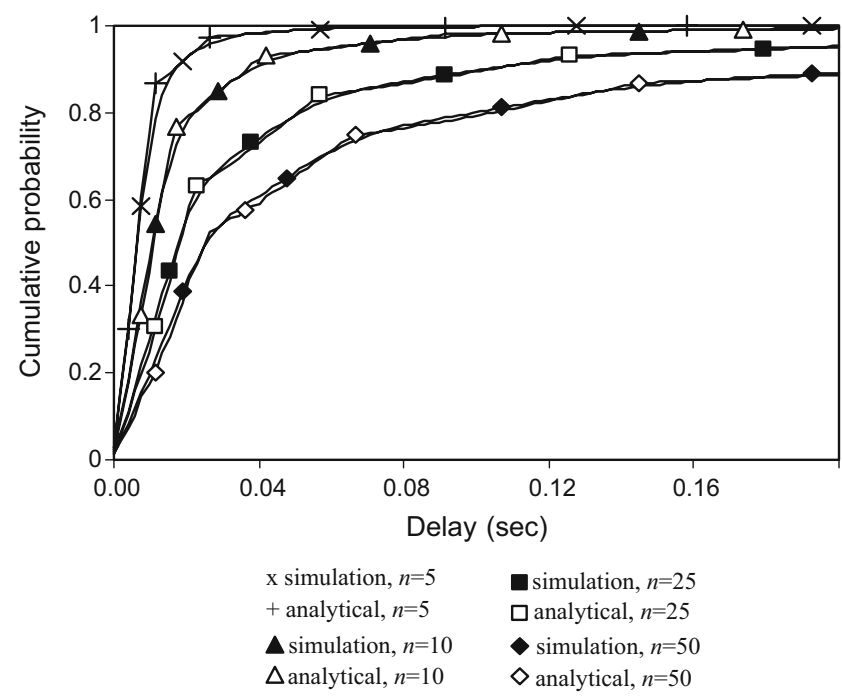

Figure 5 Cumulative delay distribution for RTS/CTS access mode for different network sizes 
Table 1 System parameter values

\begin{tabular}{ll}
\hline MAC header & 224 bits at $11 \mathrm{Mbit} / \mathrm{s}$ \\
Physical header PHY & $192 \mathrm{bits}$ at $1 \mathrm{Mbit} / \mathrm{s}$ \\
ACK & $112 \mathrm{bits}$ at $11 \mathrm{Mbit} / \mathrm{s}+\mathrm{PHY}$ \\
RTS & $160 \mathrm{bits}$ at $1 \mathrm{Mbit} / \mathrm{s}+\mathrm{PHY}$ \\
CTS & $112 \mathrm{bits}$ at $1 \mathrm{Mbit} / \mathrm{s}+\mathrm{PHY}$ \\
Propagation delay, $\delta$ & $1 \mu \mathrm{s}$ \\
Slot time, $\sigma$ & $20 \mu \mathrm{s}$ \\
SIFS & $10 \mu \mathrm{s}$ \\
DIFS & $50 \mu \mathrm{s}$ \\
Minimum $C W, W$ & 32 \\
Number of $C W$ sizes, $m$ & 5 \\
Retransmission limit, $R$ & 6
\end{tabular}

$1 \mathrm{Mbit} / \mathrm{s}$, the control frames and the payload data are transmitted at $1 \mathrm{Mbit} / \mathrm{s}$; when $C=11 \mathrm{Mbit} / \mathrm{s}$, the control frames are transmitted at rates as shown at Table 1 and the payload data at $11 \mathrm{Mbit} / \mathrm{s}$. In this section we present analytical results only for basic access as the results of RTS/ CTS scheme are similar to those of basic access.

Figure 6 explores the effect of network and initial contention window sizes on packet collision and drop probabilities. The figure demonstrates that both the packet collision and drop probabilities are highly dependent on the number of stations since a large network size results in a higher collision probability and, thus, significant performance degradation. Figure also indicates that increasing initial contention window size results in a significant decrease on packet collision and drop probabilities, especially for large networks.

Figure 7 plots average packet delay and delay jitter as a function of the number of stations $n$ for various initial contention window values. High $W$ values result in slightly lower average packet delay and jitter values for small

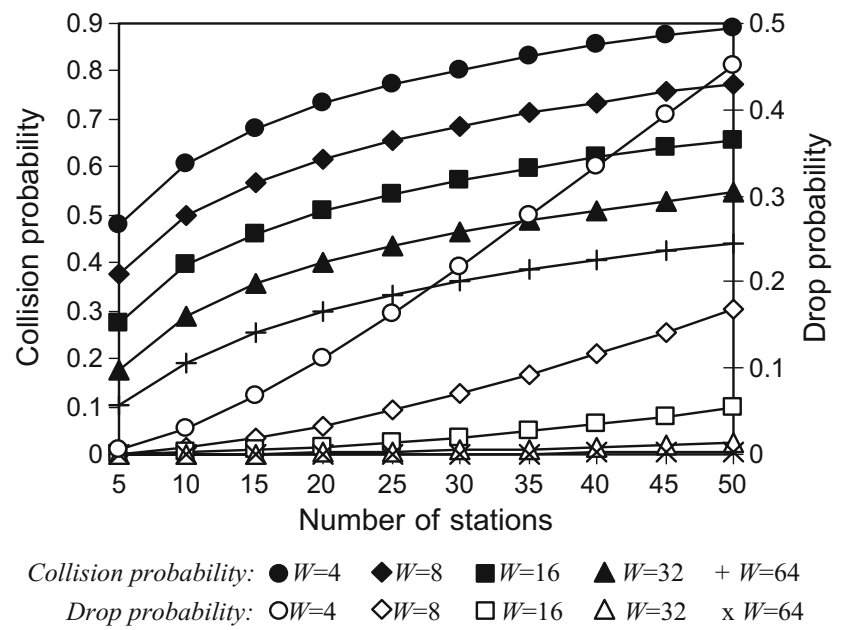

Figure 6 Collision and drop probabilities versus number of stations

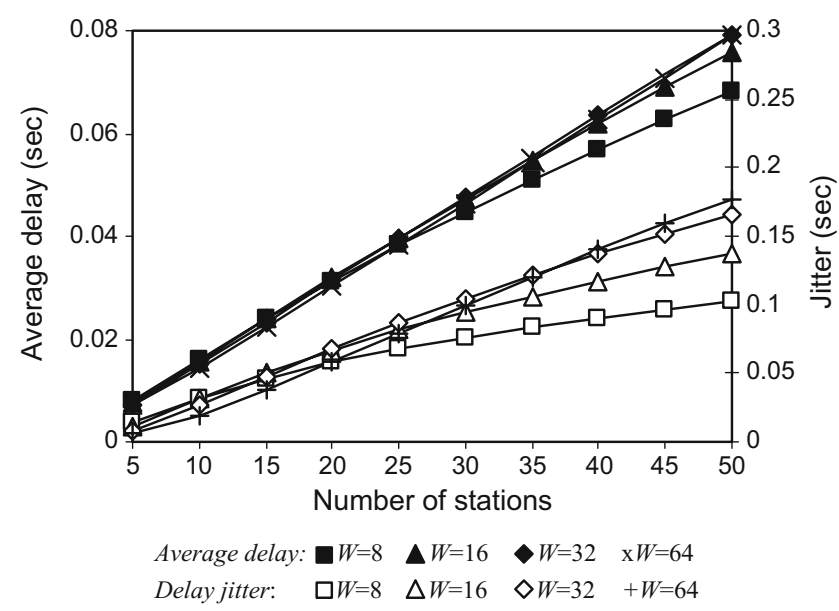

Figure 7 Average delay and delay jitter versus number of stations

networks but low $W$ values result in a considerable decrease on both average delay and jitter values for large networks.

However, as Fig. 6 indicates, low $W$ values result in high packet drop probability values especially for high $n$. As a conclusion, Figs. 6 and 7 indicate that the optimum $W$ value should be adequately low in order to reduce packet delay and jitter but high enough in order to result in an acceptable packet drop probability which is application dependent.

Figure 8 plots average delay and jitter versus packet length for network sizes $n=5,25$ and initial contention window values $W=16,32$. The figure shows that the average delay and the jitter increase as the packet length increases. The average delay is independent of $W$ for small $(n=5)$ and medium $(n=25)$ network sizes. For $n=5$ the jitter is lower for $W=32$ than $W=16$ due to the fact that the collision probability for $W=32$ is lower than $W=16$, thus, stations with $W=32$ utilize more the low backoff stages for successful transmissions resulting in reduced delay vari-

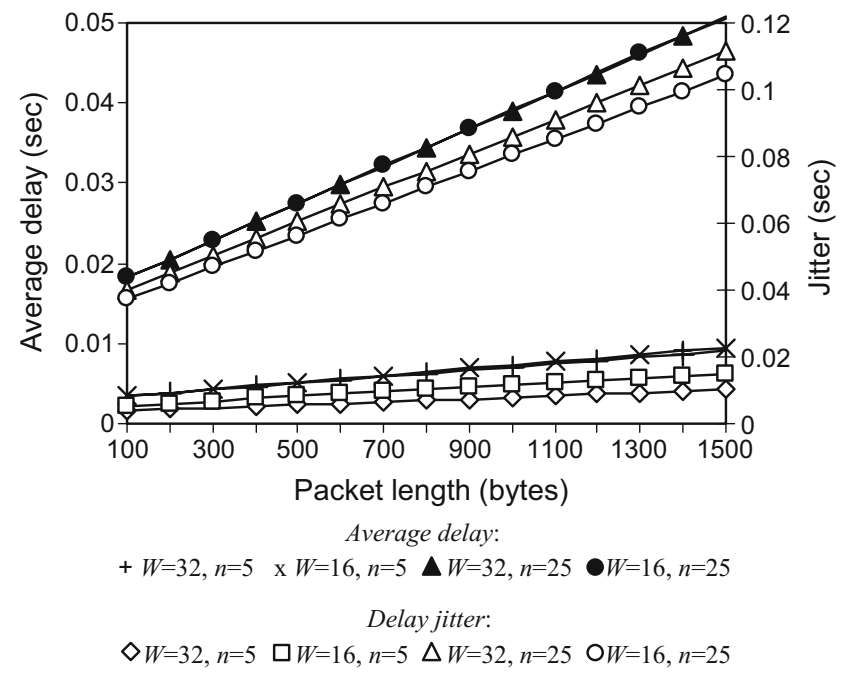

Figure 8 Average delay and jitter versus packet length 
ability (jitter). For medium network sizes the jitter gets higher for $W=32$ than $W=16$. Although the collision probability for $W=32$ is lower than $W=16$, the stations with $W=16$ use more evenly the stages for successful transmissions where as stations with $W=32$ use the lower stages with higher probability, and, as the average delay is increased, even usage of stages for $W=16$ results in lower variability in delays and, thus, lower jitter.

Figure 9 plots the average delay and jitter versus $m$ (number of different contention window sizes) for initial contention window $W=32, R=6$ and different network sizes. For small network size average delay and jitter are insensitive $m$. For medium and large network sizes $(n=25$ and $n=50$, respectively) average delay degrades as $m$ increases because the collision probability attains lower values. For medium and large network sizes the jitter increases for higher values of $m$ since the stations select backoff values using larger window sizes resulting in increased delay variability and, thus, jitter.

Figure 10 depicts average delay and jitter versus initial contention window for various network sizes. The average delay for small and medium size networks is independent of $W$ (consistent with Fig. 7). Although someone should expect high delay values for $W=4$ and $n=25,50$ that provides high number of collided packets and consequently high number of dropped packets (Fig. 6), the delay values remain low. This happens because the dropped packets are not included in the calculation of the average delay thus the successfully transmitted packets provide low delays. For $W=512$ and $n=25$ the collision and the drop probability are low so very low delays are expected, but the number of empty slots is high so the delays stay mostly unchanged. For $W=32$ and $n=25$ there is a balance in provided delays between the drop probability and the empty slots, so the average delay stay unaffected. On the other hand, the jitter for small networks decreases as $W$ increases because the

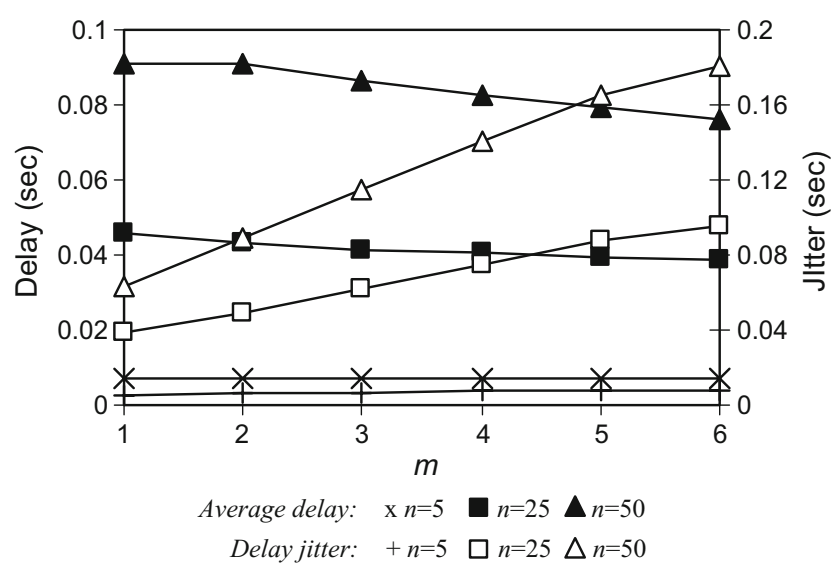

Figure 9 Average delay and jitter versus number of contention windows $m$

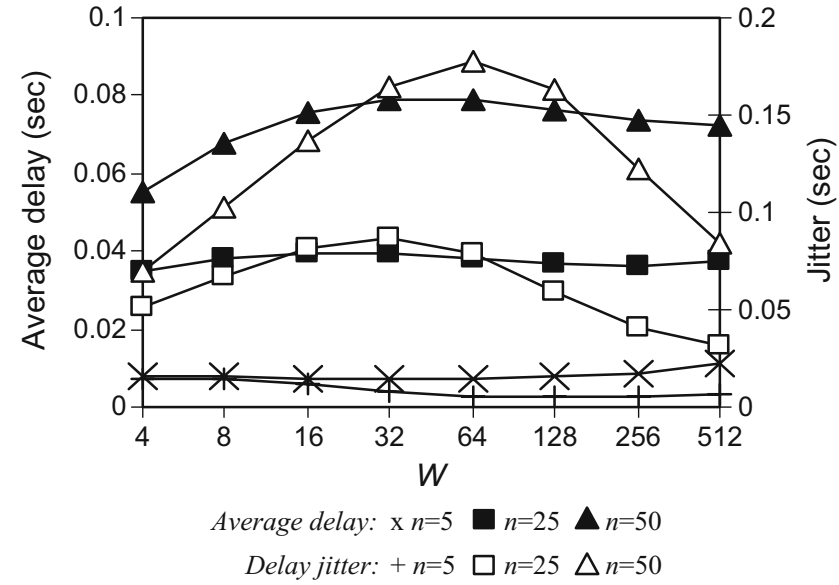

Figure 10 Average delay and jitter versus initial contention window

collision probability is low and the stations utilize only low backoff stages. For medium and large network sizes the jitter gets higher (up to a value) as the $W$ increases since stations utilize more the lower stages resulting in increased delay variability. Conversely, for high $W$ sizes the jitter degrades since the stations utilize only the low stages (almost exclusively stage $0,1,2$ for $W=512$ ) resulting low variability of delays and, thus, jitter. The standard proposed value of $32(W=32)$ results in high jitter values for medium and large networks.

\section{Conclusions}

In this paper we present a complete delay analysis that computes the average packet delay per stage, the average packet drop time, the average packet delay, the packet delay jitter, and the packet delay distribution of IEEE 802.11 DCF WLANs. The delay distribution model computes the probability of a packet to be successfully transmitted with delay time lower than a given value. In order to calculate the defer time of a station in jitter and delay distribution models, we assume that delay variation mainly originates from different backoff values selected at different stages and not from different time variations of backoff slots. This approximation increases computational efficiency and provides accurate analytical results.

The developed analytical models are simple, efficient and fairly accurate since they are validated by comparing analytical against simulation results. Results show that delay jitter (a) highly depends on collisions the packets suffer, and (b) it does not always follow average delay's increases and decreases, (c) very lower $(W=4,8)$ and very higher $(W=256,512)$ values than standard value $(W=32)$ of initial contention window result in low jitter values. Results demonstrate that high number of different contention 
window sizes (for ex. $m=6$ ) provide high jitter values which suggest that smaller values of $m$ may be more appropriate specially if real-time stations are to be used. Average delay increases as number of stations, and packet length increase and decreases as the number of contention window sizes increase. Jitter increases as number of stations, packet length, and number of contention window sizes increase. Finally, the proposed jitter model gives results that are closer to simulation results compared to the jitter model presented in [13] that computes the average defer time at each backoff stage.

Future work could include the application of the proposed models to guarantee Quality of Service (QoS) for real-time applications in WLANs. More specifically, the presented performance metrics could be applied to develop admission control algorithms for admitting voice, video and data calls over WLANs. Another possible direction for future work could be the extension of the proposed analysis to IEEE $802.11 \mathrm{e}$ and $802.11 \mathrm{n}$ protocols.

Acknowledgement The project is co-funded by the European Social Fund \& National Resources- EPEAEK II-ARCHIMIDIS.

\section{References}

1. IEEE standard for Wireless LAN Medium Access Control (MAC) and Physical Layer (PHY) specifications (2001) IEEE Std: 802.11b-1999/Cor 1-2001

2. Bianchi G (2000) Performance analysis of the IEEE 802.11 distributed coordination function. IEEE J Sel Areas Commun 18 (3):535-547. doi:10.1109/49.840210

3. Wu H, Peng Y, Long K, Cheng S, Ma J (2002) "Performance of reliable transport protocol over IEEE 802.11 Wireless LAN: analysis and enhancement". In Proceedings of IEEE INFOCOM 599-607

4. Cali F, Conti M, Gregori E (2000) Dynamic tuning of the IEEE 802.11 protocol to achieve a theoretical throughput limit. IEEE/ ACM T Network 8(6):785-790. doi:10.1109/90.893874

5. Chhaya HS, Gupta S (1997) Performance modelling of asynchronous data transfer methods of IEEE 802.11 MAC protocol. Wirel Netw 3(3):217-234. doi:10.1023/A:1019109301754
6. Hadzi-Velkov Z, Spasenovski B (2003) "Saturation throughputdelay analysis of IEEE 802.11 DCF in fading channel". In Proceedings of IEEE International Conference on Communications (ICC) 1:121-126

7. Chatzimisios P, Boucouvalas AC, Vitsas V (2007) Achieving performance enhancement in IEEE 802.11 WLANs by using the DIDD backoff mechanism. Int J Commun Syst 20(1):23-41. doi:10.1002/dac. 811

8. Chatzimisios P, Boucouvalas AC, Vitsas V (2003) "IEEE 802.11 Packet delay - A finite retry limit analysis". In Proceedings of IEEE GLOBECOM 2:950-954

9. Carvalho MM, Garcia-Luna-Aceves JJ (2003) "Delay Analysis of IEEE 802.11 in single-hop networks". In Proceedings of 11th IEEE International Conference on Network Protocols (ICNP) 146-155

10. Vukovic I, Smavatkul N (2004) "Delay analysis of different backoff algorithms in IEEE 802.11". In Proceedings of IEEE Vehicular Technology Conference (VTC '04-Fall) 6:4553-4557, September

11. Raptis P, Vitsas V, Paparrizos K, Chatzimisios P, Boucouvalas AC, Adamidis P. (2005) "Packet delay modeling of IEEE 802.11 Wireless LANs". In Proceedings of 2nd International Conference on Cybernetics and Information Technologies, Systems and Applications (CITSA) I:71-76

12. Raptis P, Vitsas V, Paparrizos K, Chatzimisios P, Boucouvalas AC (2005) "Packet delay distribution of the IEEE 802.11 distributed coordination function". In Proceedings of 6th IEEE International Symposium on a World of Wireless, Mobile and Multimedia Networks (WOWMOM), 299-304

13. Li M, Claypool M, Kinicki R (2006) "Packet dispersion in IEEE 802.11 wireless networks". In Proceedings 31st IEEE Conference on Local Computer Networks (LCN) 721-729

14. Zhai H, Fang Y (2003) "Performance of wireless LANs Based on IEEE 802.11 MAC protocols". In Proceedings of 14th IEEE Symposium on Personal, Indoor and Mobile Radio Communication (PIMRC) 2586-2590

15. Tickioo O, Sikdar B (2004) "Queuing analysis and delay mitigation in IEEE 802.11 Random Access MAC based Wireless Networks". In Proceedings of IEEE INFOCOM 2:1404-1413

16. Banchs A, Serrano P, Azcorra A (2006) End-to-end delay analysis and admission control in 802.11 DCF WLANs. Comput Commun 29(7):842-854. doi:10.1016/j.comcom.2005.08.006

17. Raptis P, Banchs A, Vitsas V, Paparrizos K, Chatzimisios P (2006) "Delay distribution analysis of the RTS/CTS mechanism of IEEE 802.11". In Proceedings of the 31st IEEE Conference on Local Computer Networks (LCN) 404-410

18. Raptis P, Banchs A, Paparrizos K (2006) "A simple and effective delay distribution analysis for IEEE 802.11". In Proceedings of 17th IEEE Symposium on Personal, Indoor and Mobile Radio Communications (PIMRC) 1-5 